\title{
Development and Evaluation of an iiPCR Assay for Salmonella and Shigella Detection on a Field-Deployable PCR System
}

\author{
Tian Du, ${ }^{1}$ Ji-hong Lin, ${ }^{2}$ Jun-hua Zhao, ${ }^{3}$ Hai-bo Wang $\mathbb{D}^{3},{ }^{3}$ and Qiu-hua Mo ${ }^{3}{ }^{3}$ \\ ${ }^{1}$ Futian District Center for Disease Control and Prevention, Shenzhen 518040, Guangdong, China \\ ${ }^{2}$ Zhongshan International Travel Healthcare Center, Zhongshan 528400, Guangdong, China \\ ${ }^{3}$ State Key Laboratory of Diarrhea Disease Detection, Zhuhai International Travel Healthcare Center, Zhuhai 519020, \\ Guangdong, China
}

Correspondence should be addressed to Hai-bo Wang; wanghb1013@hotmail.com and Qiu-hua Mo; zhithc-lab@hotmail.com

Received 14 April 2020; Revised 7 August 2020; Accepted 27 August 2020; Published 7 September 2020

Academic Editor: Cinzia Marianelli

Copyright (c) 2020 Tian Du et al. This is an open access article distributed under the Creative Commons Attribution License, which permits unrestricted use, distribution, and reproduction in any medium, provided the original work is properly cited.

\begin{abstract}
Background. Salmonella and Shigella are often associated with fecal-oral transmission and cause large-scale outbreaks in centralized catering units and, therefore, should be frequently and strictly monitored, especially among food handlers. However, no specific and sensitive on-site detection method is available until now. Methods. In this study, an insulated isothermal PCR assay for the detection of Salmonella and Shigella on a field-deployable PCR system was developed. Specificity, sensitivity, reproducibility, and clinical accuracy of the assay were characterized and evaluated. Results. The insulated isothermal PCR assay could be completed within 58 minutes with minimal pretreatment needed. The assay was specific and with good reproducibility. The limit of detection was $10^{3} \mathrm{CFU} / \mathrm{mL}$ and $10^{1} \mathrm{CFU} / \mathrm{mL}$ for Salmonella and Shigella, respectively, which was comparable to multiplex real-time PCR. Mock on-site clinical evaluation results showed that the analytical sensitivity and specificity of the insulated isothermal PCR assay were $100 \%$ and $96.6 \%$, while the positive predictive value and negative predictive value were $94.1 \%$ and $100 \%$, respectively. Conclusion. Based on our results, we believe that the assay developed herein could serve as an alternative method for preliminary screening and provide a valuable platform for the on-site detection of Salmonella and Shigella, especially in resource-limited and developing countries.
\end{abstract}

\section{Introduction}

Diarrhea is an ordinary disease but with a relatively high morbidity and mortality in developing countries according to recent reports $[1,2]$. Once infected, patients may suffer from the symptoms for days and spread the disease to others, which caused high socioeconomic burden [3]. Generally, the causative agent for infectious diarrhea may be viruses (such as norovirus and rotavirus), parasites (such as Giardia lamblia and Entamoeba histolytica), or bacteria (such as Salmonella and Shigella). Among these causative agents, Salmonella and Shigella are often associated with fecal-oral transmission and cause large-scale outbreaks in centralized catering units $[4,5]$. Therefore, as a preventive action, governments issued laws for obligatory Salmonella and Shigella screening among food handlers.
The gold standard for the screening and detection of Salmonella and Shigella is culture. However, many hands-on steps are needed which make the method labor-intensive and time-consuming. Moreover, the lower limit of detection (LOD) of the method may be as high as $10^{4} \mathrm{CFU} / \mathrm{mL}$ [6]. With the aim of improving the effectiveness and sensitivity, we developed several nucleic acid-based assays, including PCR $[6,7]$, DNA microarray [8], and real-time nucleic acid sequence-based amplification (RT-NASBA) [9], for the detection of Salmonella and Shigella or other diarrhea-related pathogens. However, these assays are not suitable for on-site detection, where no sophisticated thermal cycler is available. Zhuang et al. developed the loop-mediated isothermal amplification (LAMP) assay for the on-site detection of Salmonella [10], while false-positive results may be generated due to the fact that the detection of 
LAMP products was via either turbidity or generic fluorescent dyes.

Recently, a fluorescent probe hydrolysis-based insulated isothermal PCR (iiPCR) technology was invented and commercially available [11]. With the field-deployable PCR machine (POCKIT Nucleic Acid Analyzer, GeneRadar Biotechnology Corp., Xiamen, China), on-site detection becomes possible. In our previous study, we have established a reverse transcription-iiPCR method for rapid on-site detection of yellow fever virus. The detection can be completed within one hour automatically with analytical specificity and sensitivity up to $100 \%$ and $92.31 \%$, respectively [12]. In the current study, an iiPCR assay for on-site detection of Salmonella and Shigella on this field-deployable PCR system was developed and its specificity, sensitivity, and reproducibility were evaluated. Stool samples were used to evaluate the accuracy of the assay for its potential clinical application.

\section{Materials and Methods}

2.1. Samples. Human stool samples were collected using a stool tube from food handlers who came to our center for obligatory Salmonella and Shigella screening during 2017-2019 and first diagnosed by the bacterial culture method as described previously [6]. 183 samples, including 36 samples positive for Salmonella, 28 samples positive for Shigella, and 119 negative samples, were selected randomly. These samples were then used to evaluate the iiPCR assay. The study was approved by the ethics committee of the Zhuhai International Travel Healthcare Center and in compliance with the Helsinki Declaration. Informed consent was obtained from each study participant.

2.2. Primers and Probes. Primers and probes used in the current study are listed in Table 1 . Before designing the primers and probes, relevant sequences of Salmonella and Shigella in the GenBank were aligned and the conserved ssaR gene and $i p a H$ gene were selected, respectively. The primers and probe sequences were generated using PrimerSelect software and further aligned with other pathogens, including Vibrio, Staphylococcus, and Campylobacter, to avoid the possibility of any undesired match. The probes were designed according to the principles recommended for the iiPCR (http://www.iipcr.com/index.php?func=faq), including moderate GC content and shorter length. The amplicons were analyzed with the MFold program (http://unafold.rna. albany.edu/?q=mfold) to avoid those amplicons with major secondary structures.

2.3. The iiPCR Assay. After several rounds of optimization through adjusting the concentration of buffer, primers, and probes, the iiPCR was established as follows. The reaction mixture, in R-Tube (GeneRadar Biotechnology Corp., Xiamen, China), contained $25.0 \mu \mathrm{L}$ Uni-ii buffer A, $1.5 \mu \mathrm{L}$ iiPCR Taq DNA Polymerase $(5 \mathrm{U} / \mu \mathrm{L}), 0.25 \mu \mathrm{L}$ tetramethylene sulfoxide, $0.5 \mu \mathrm{L}$ trehalose, $0.6 \mu \mathrm{M}$ of each primers (Sal-F, Sal-R, Shi-F, Shi-R), $0.3 \mu \mathrm{M}$ of each probes (Sal-P, Shi-P), and $5 \mu \mathrm{L}$ DNA template. After a short centrifugation on cubee $^{\mathrm{TM}}$ microcentrifuge (GeneRadar Biotechnology Corp., Xiamen, China), the R-Tube was loaded onto the POCKIT device. A default program of $50^{\circ} \mathrm{C}$ for $10 \mathrm{~min}$ and $95^{\circ} \mathrm{C}$ for $48 \mathrm{~min}$ was conducted. Fluorescent signals were collected and signal-to-noise $(\mathrm{S} / \mathrm{N})$ ratios were calculated through dividing signals collected after iiPCR by those from before iiPCR [11]. Results were displayed on the screen automatically as "+" (Positive), “-” (Negative), or "?” (Unknown), using the default $\mathrm{S} / \mathrm{N}$ thresholds.

2.4. Characterization of the Assay. Before specificity, sensitivity, and reproducibility evaluation, Salmonella (AB91111) and Shigella (AB200061) were cultured overnight in nutrient broth (LandBridge, Beijing, China) at $36^{\circ} \mathrm{C}$ and harvested for concentration determination by plate counting technique on XLD as described previously [13]. The specificity of the iiPCR assay was evaluated by detection of Salmonella, Shigella, and other related pathogens, including Vibrio parahaemolyticus, Staphylococcus aureus, norovirus GII, rotavirus A, Giardia lamblia, Campylobacter jejuni, Yersinia enterocolitica, and Vibrio cholera O1. The sensitivity of the iiPCR assay was evaluated by serial diluted Salmonella and Shigella and expressed as the limit of detection (LOD). The reproducibility of the iiPCR assay was evaluated through detection of $10^{6} \mathrm{CFU} / \mathrm{mL}$ (high concentration) and $10^{4} \mathrm{CFU} / \mathrm{mL}$ (low concentration) of both Salmonella and Shigella standard strains five times by two different operators.

2.5. Mock On-Site Evaluation of the Assay. 183 samples (including both positive and negative samples as diagnosed by bacterial culture) were randomly selected and labeled blindly. To mimic on-site detection, a soybean-sized stool sample was first resuspended in $0.5 \mathrm{~mL}$ buffered saline, stood at room temperature for $5 \mathrm{~min}$, and the supernatant was then boiled at $100^{\circ} \mathrm{C}$ for $5 \mathrm{~min}$, which was used as the template for iiPCR. To minimize the heterogeneity problem of the samples, adjacent soybean-sized stool samples were collected for culture simultaneously. The analytical sensitivity and specificity, as well as the positive predictive value (PPV) and negative predictive value (NPV), were calculated as described previously [14].

\section{Results}

3.1. Characterization of the iiPCR Assay. After establishment, we evaluated the specificity and sensitivity of the iiPCR assay. Both evaluations performed in triple and 100\% detection endpoints were shown. As shown in Figures 1(a) and 1(b), only Salmonella and Shigella could be detected at the $520 \mathrm{~nm}$ and $550 \mathrm{~nm}$ channel, respectively, while Vibrio parahaemolyticus and Staphylococcus aureus could not be detected. Other pathogens, including norovirus GII, rotavirus A, Giardia lamblia, Campylobacter jejuni, Yersinia enterocolitica, and Vibrio cholera O1, were also negative (data not shown). These results indicated the high specificity of the iiPCR assay. The sensitivity (as shown by LOD) of the 
TABLe 1: Primers and probes used in the iiPCR assay.

\begin{tabular}{lc}
\hline Primer/probe & Sequence $\left(5^{\prime}-3^{\prime}\right)$ \\
\hline Sal-F & GCT TGT ACT TTC CTT ATT C \\
Sal-R & AGY GYC AGC GAC CTG AAC \\
Sal-P & FAM-ACG CTA TTA GCT GTA AAA GAG CGC TG-BHQ1 \\
Shi-F & TGA AGG AAA TGC GTT TCT \\
Shi-R & ACT TCT GAC CAT GGC TTC \\
Shi-P & HEX-TGT CGG GAG TGA CAG CAA ATG ACC TCC GCA-BHQ1 \\
\hline
\end{tabular}

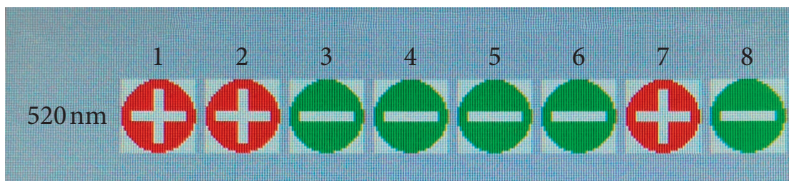

(a)

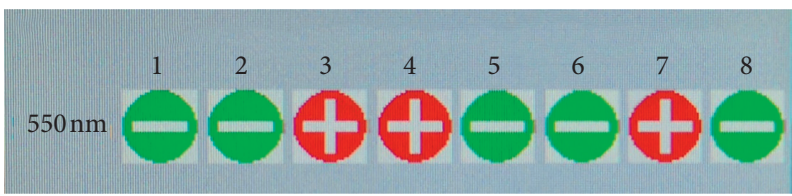

(b)

FIgURE 1: Representative results displayed during specificity evaluation. (a) and (b) Specificity analysis for Salmonella and Shigella, respectively. Samples 1 to 8 were Salmonella (AB91111, AB91018), Shigella (AB200061, AB200060), Vibrio parahaemolyticus (ATCC17802), Staphylococcus aureus (ATCC25923), positive control, and negative control.

iiPCR assay was determined by detecting serially diluted Salmonella and Shigella standard strains. As shown in Figures 2(a) and 2(b), the LOD for Salmonella and Shigella was $10^{3} \mathrm{CFU} / \mathrm{mL}$ and $10^{1} \mathrm{CFU} / \mathrm{mL}$, respectively. The reproducibility of the iiPCR assay was evaluated through repeated detection of both high and low concentration of Salmonella and Shigella. Results showed that Salmonella and Shigella could be detected in all ten tests, suggesting good reproducibility of the assay (data not shown).

3.2. Mock On-Site Evaluation of the iiPCR Assay. In mock onsite evaluation using 183 stool samples, a total of 36 samples positive for Salmonella and 28 samples positive for Shigella were detected by both iiPCR assay and culture. However, four samples which were positive by iiPCR assay were tested to be negative by culture. Therefore, using culture as the gold standard, the calculated analytical sensitivity and specificity of the iiPCR assay were $100 \%$ and $96.6 \%$, while the PPV and NPV were $94.1 \%$ and $100 \%$, respectively (Table 2).

\section{Discussion}

Since Salmonella and Shigella are fecal-oral transmitted and cause large-scale outbreaks in centralized catering units, it is of great importance to frequently and strictly monitor food handlers. However, no specific and sensitive on-site detection method is available until now. In the current study, an iiPCR assay for the detection of Salmonella and Shigella on a field-deployable PCR system was developed and evaluated.

The specificity, sensitivity, and reproducibility characterization of the iiPCR assay were performed. To obtain high specificity, the primers/probes were designed within the most conserved regions of the genome of Salmonella and Shigella and further aligned with other related pathogens.
Therefore, the iiPCR assay showed great selective ability for Salmonella and Shigella without cross-reaction with Vibrio parahaemolyticus, Staphylococcus aureus, norovirus GII, rotavirus A, Giardia lamblia, Campylobacter jejuni, Yersinia enterocolitica, and Vibrio cholera O1 (Figure 1). The sensitivity (as shown by LOD) for Salmonella and Shigella was $10^{3} \mathrm{CFU} / \mathrm{mL}$ and $10^{1} \mathrm{CFU} / \mathrm{mL}$, respectively. This result is comparable to both our and other previous reports. In our previous study, the LOD for Salmonella and Shigella was $10^{3} \mathrm{CFU} / \mathrm{mL}$ and $10^{1} \mathrm{CFU} / \mathrm{mL}$, respectively [6]. In other duplex assays, Cunningham et al. reported LOD of $990 \mathrm{CFU} /$ $\mathrm{mL}$ and $52 \mathrm{CFU} / \mathrm{mL}$, while Van Lint et al. reported LOD of $5528 \mathrm{CFU} / \mathrm{mL}$ and $61 \mathrm{CFU} / \mathrm{mL}$ for Salmonella and Shigella, respectively $[15,16]$. During reproducibility characterization, Salmonella and Shigella could be detected in repeated tests by different operators, suggesting good reproducibility of the assay, which was an important parameter for clinical usage. When compared to the culture method using a national standard (which was also similar to standard ISO procedures), there was no difference with regard to specificity and reproducibility. However, the LOD of the culture method was $10^{4} \mathrm{CFU} / \mathrm{mL}$ and $10^{3} \mathrm{CFU} / \mathrm{mL}$ for Salmonella and Shigella, respectively [6], which was much higher than the iiPCR assay.

The mock on-site evaluation of the iiPCR assay was conducted using stool samples. The assay showed extremely high analytical sensitivity, specificity, PPV, and NPV, which indicated that the assay could be used as an efficient tool for the detection of Salmonella and Shigella. The four samples which were iiPCR positive and culture negative may contain viable but nonculturable (VBNC) Salmonella, as indicated by previous reports [17], or contain dead microorganisms. Temperature shift methods, including $56^{\circ} \mathrm{C}$ for $15 \mathrm{sec}, 37^{\circ} \mathrm{C}$ for 3 days, or $30^{\circ} \mathrm{C}$ for 2 days, as indicated by previous studies [18-20], had been applied; however, they were still could not be cultured. As resuscitation-promoting stimuli (pyruvate 


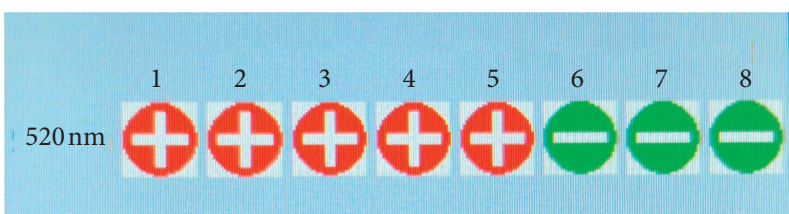

(a)

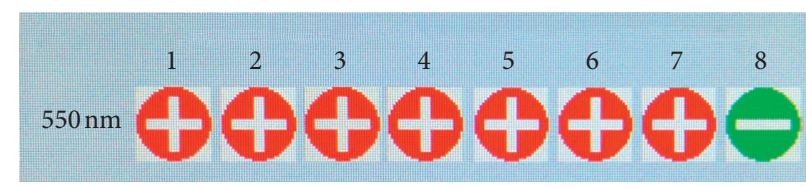

(b)

Figure 2: Representative results displayed during sensitivity evaluation. (a) and (b) Sensitivity analysis for Salmonella and Shigella, respectively. Samples 1 to 8 were Salmonella and Shigella from $10^{7} \mathrm{CFU} / \mathrm{mL}$ to $10^{0} \mathrm{CFU} / \mathrm{mL}$.

TABle 2: Clinical evaluation of the iiPCR assay.

\begin{tabular}{lccc}
\hline iiPCR & \multicolumn{3}{c}{ Bacterial culture } \\
& Positive & Negative & Total \\
\hline Positive & 64 & 4 & 68 \\
Negative & 0 & 115 & 115 \\
Total & 64 & 119 & 183 \\
\hline
\end{tabular}

and $\alpha$-ketobutyrate), as suggested by Morishige et al. [21], were not available currently, whether they were in real VBNC state or just dead microorganisms needed further investigation. If some cases with clear clinical symptoms showed negative culture results, other serological tests should be conducted to confirm the results.

There are several advantages of this iiPCR assay. Unlike the PCR assay which needs a complicated and time-consuming nucleic acid extraction step, the iiPCR assay needs minimal pretreatment for samples (resuspend and boil), which is eligible for on-site detection. Moreover, lyophilized reagents, which were formatted for easy shipping at ambient temperature and storage, were used during the assay, and the field-deployable PCR machine (31-26-15 cm (W-D-H); $2.1 \mathrm{~kg}$ ) can be packed into a suitcase and operated using a rechargeable battery (detailed information could be obtained from http://www.genereach.com). Since everything is packed, the detection could be taken place anytime and anywhere, which is highly suitable in resource-limited areas where conventional PCR facilities are lacking and in developing countries where stable power supply is not available. Furthermore, since the iiPCR was used to detect nucleic acid, theoretically it could effectively detect the nucleic acid from any kinds of samples, including food or environmental samples [22]. All these advantages made the iiPCR assay an easy-to-use tool for the detection of Salmonella and Shigella. There were also few disadvantages of this iiPCR. Firstly, now the iiPCR system is a qualitative system without quantitative module, which could not give the absolute quantification of the pathogens detected. However, its optical system is similar to the real-time PCR machine as stated by the producer. Therefore, it has the potential to be upgraded to a quantitative system in the near future. Secondly, the iiPCR, similar to other nucleic acid detection methods, could only detect targeted bacteria (Salmonella and Shigella in the current assay), while other bacteria which could also cause diarrhea are ignored [23]. Thirdly, the assay could not distinguish live or dead microorganisms as indicated in the current study. However, as an alternative method for preliminary screening, the iiPCR is acceptable. If the result is positive, other tests could be further conducted for confirmation.

\section{Conclusion}

In conclusion, an iiPCR assay for the detection of Salmonella and Shigella on a field-deployable PCR system was developed successfully. Our results showed that the assay was specific, sensitive, and reproducible, which would serve as an alternative method for preliminary screening and provide a valuable platform for the on-site detection of Salmonella and Shigella, especially in resource-limited and developing countries.

\section{Data Availability}

The datasets generated during and/or analyzed during the current study are available from the corresponding author on reasonable request.

\section{Disclosure}

The funders had no role in the study design, data collection, and interpretation or in the decision to submit the work for publication.

\section{Conflicts of Interest}

The authors have declared that no conflicts of interest exist.

\section{Authors' Contributions}

All authors participated in the design and implementation, analysis and interpretation of the results, and the development of the manuscript. All authors had full access to the data and gave final approval before submission.

\section{Acknowledgments}

This work was supported by the Public Welfare Scientific Research Project of Health in Futian District, Shenzhen, China (grant \#FTWS2020067), the Science and Technology Program of Guangdong, China (grant \#2018B020207013), and the Science and Technology Programs of General Administration of Customs, China (grant \#2018IK047). 


\section{References}

[1] R. C. Reiner Jr., N. Graetz, D. C. Casey et al., "Variation in childhood diarrheal morbidity and mortality in Africa, 2000-2015," New England Journal of Medicine, vol. 379, no. 12, pp. 1128-1138, 2018.

[2] I. A. Blacker, C. Troeger, B. F. Blacker et al., "Morbidity and mortality due to shigella and enterotoxigenic Escherichia coli diarrhoea: the global burden of disease study 1990-2016," The Lancet Infectious Diseases, vol. 18, no. 11, pp. 1229-1240, 2018.

[3] F. Ngabo, M. Mvundura, L. Gazley et al., "The economic burden attributable to a child's inpatient admission for diarrheal disease in Rwanda," PLoS One, vol. 11, no. 2, Article ID e0149805, 2016.

[4] M. Muvhali, A. M. Smith, A. M. Rakgantso, and K. H. Keddy, "Investigation of Salmonella Enteritidis outbreaks in South Africa using multi-locus variable-number tandem-repeats analysis, 2013-2015," BMC Infectious Diseases, vol. 17, no. 1, p. 661, 2017.

[5] V. Rew, P. Mook, S. Trienekens et al., "Whole-genome sequencing revealed concurrent outbreaks of shigellosis in the English Orthodox Jewish Community caused by multiple importations of Shigella sonnei from Israel," Microbial Genomics, vol. 4, no. 3, 2018.

[6] X.-J. Tang, Z. Yang, X.-B. Chen, W.-F. Tian, C.-N. Tu, and H.-B. Wang, "Verification and large scale clinical evaluation of a national standard protocol for Salmonella spp./Shigella $s p p$. screening using real-time PCR combined with guided culture," Journal of Microbiological Methods, vol. 145, pp. 14-19, 2018.

[7] H.-B. Wang, Q.-H. Mo, Q. Wang et al., "Probe-free and sensitive detection of diarrhea-causing pathogens using RTPCR combined high resolution melting analysis," Biologicals, vol. 44, no. 5, pp. 360-366, 2016.

[8] H. Sun, Q.-H. Mo, J.-C. Lin et al., "Rapid simultaneous screening of seven clinically important enteric pathogens using a magnetic bead based DNA microarray," World Journal of Microbiology and Biotechnology, vol. 27, no. 1, pp. 163-169, 2011.

[9] Q.-H. Mo, H.-B. Wang, H.-R. Dai et al., "Rapid and simultaneous detection of three major diarrhea-causing viruses by multiplex real-time nucleic acid sequence-based amplification," Archives of Virology, vol. 160, no. 3, pp. 719-725, 2015.

[10] L. Zhuang, J. Gong, Q. Li et al., "Detection of Salmonella spp. by a loop-mediated isothermal amplification (LAMP) method targeting bcfD gene," Letters in Applied Microbiology, vol. 59, no. 6, pp. 658-664, 2014.

[11] Y.-L. Tsai, H.-C. Wang, C.-F. Lo et al., "Validation of a commercial insulated isothermal PCR-based POCKIT test for rapid and easy detection of white spot syndrome virus infection in Litopenaeus vannamei," PLoS One, vol. 9, no. 3, Article ID e90545, 2014.

[12] J.-H. Zhao, W.-G. Li, T. Du et al., "A reverse transcriptioninsulated isothermal PCR method for the detection of yellow fever virus," Chinese Journal of Frontier Health and Quarantine, vol. 42, no. 4, pp. 229-232, 2019.

[13] M. Srisawat and W. Panbangred, "Efficient and specific detection of Salmonella in food samples using astn-based loopmediated isothermal amplification method," BioMed Research International, vol. 2015, Article ID 356401, 7 pages, 2015.

[14] E.-J. Yoon, E. H. Lee, D. H. Hwang, H. Lee, J.-H. Baek, and S. H. Jeong, "Direct detection of intact Klebsiella pneumoniae carbapenemases produced by enterobacterales using MALDI-
TOF MS," Journal of Antimicrobial Chemotherapy, vol. 75, no. 5 , p. $1174,2020$.

[15] S. A. Cunningham, L. M. Sloan, L. M. Nyre, E. A. Vetter, J. Mandrekar, and R. Patel, "Three-hour molecular detection of Campylobacter, Salmonella, Yersinia, and Shigella species in feces with accuracy as high as that of culture," Journal of Clinical Microbiology, vol. 48, no. 8, pp. 2929-2933, 2010.

[16] P. Van Lint, E. De Witte, H. De Henau et al., "Evaluation of a real-time multiplex PCR for the simultaneous detection of Campylobacter jejuni, Salmonella spp., Shigella spp./EIEC, and Yersinia enterocolitica in fecal samples," European Journal of Clinical Microbiology \& Infectious Diseases, vol. 34, no. 3, pp. 535-542, 2015.

[17] J. D. Oliver, "The viable but nonculturable state in bacteria," Journal of Microbiology (Seoul, Korea), vol. 43, pp. 93-100, 2005.

[18] H. Liao, R. Zhang, K. Zhong, Y. Ma, X. Nie, and Y. Liu, "Induction of a viable but non-culturable state in Salmonella typhimurium is correlated with free radicals generated by thermosonication," International Journal of Food Microbiology, vol. 286, pp. 90-97, 2018.

[19] A. R. Gupte, C. L. E. De Rezende, and S. W. Joseph, "Induction and resuscitation of viable but nonculturable Salmonella enterica serovar typhimurium DT104," Applied and Environmental Microbiology, vol. 69, no. 11, pp. 6669-6675, 2003.

[20] N. Panutdaporn, K. Kawamoto, H. Asakura, and S.-I. Makino, "Resuscitation of the viable but non-culturable state of Salmonella enterica serovar Oranienburg by recombinant resuscitation-promoting factor derived from Salmonella Typhimurium strain LT2," International Journal of Food Microbiology, vol. 106, no. 3, pp. 241-247, 2006.

[21] Y. Morishige, K. Fujimori, and F. Amano, "Differential resuscitative effect of pyruvate and its analogues on VBNC (viable but non-culturable) Salmonella," Microbes and Environments, vol. 28 , no. 2, pp. 180-186, 2013.

[22] Y.-L. Tsai, H.-T. T. Wang, H.-F. G. Chang et al., "Development of TaqMan probe-based insulated isothermal PCR (iiPCR) for sensitive and specific on-site pathogen detection," PLoS One, vol. 7, no. 9, Article ID e45278, 2012.

[23] P. Van Lint, E. De Witte, J. P. Ursi, B. Van Herendael, and J. Van Schaeren, "A screening algorithm for diagnosing bacterial gastroenteritis by real-time PCR in combination with guided culture," Diagnostic Microbiology and Infectious Disease, vol. 85, no. 2, pp. 255-259, 2016. 